\title{
THE LTE SOLAR ABUNDANCE OF NEODYMIUM
}

\author{
A.G.A. Abdelkawy, A.M.K. Shaltout, M.M. Beheary and A. Bakry
}

Department of Astronomy and Meteorology, Faculty of Science, Al-AzharUniversity, Cairo, Egypt.Postal Code 11884.

\begin{abstract}
:
We study the solar photospheric abundance of singly ionized neodymium (Nd II) using high resolution spectroscopic data obtained by Fourier transform spectrograph (FTS). Based on the Local Thermodynamical Equilibrium (LTE) assumption, a new value of $N d$ abundance is derived. We succeed to select of 51 solar Nd II lines with accurate transition probabilities measured experimentally by Den Hartog et al. (2003) and with accurate damping parameters are determined from literature. Relying on atomic data of Opacity Distribution Function $(O D F)$, we construct theoretical photospheric solar model.The mean solar photospheric abundance obtained from all $51 \mathrm{Nd}$ II lines is $\log \varepsilon_{N d}=1.45 \pm 0.08$, which is mostly similar to the meteoric value.
\end{abstract}

Keywords: atomic data-Sun, abundances-Sun, photosphere.

\section{INTRODUCTION}

The solar chemical composition is a fundamental yardstick in astronomy but has been heavily debated in recent times. Although Nd I neutral lines are not observed in the solar photosphere, the solar photospheric spectrum does show more than 20 times of $\mathrm{Nd}$ II lines comparing with of Nd III lines. Numerous analysis show that different results have been published on the solar photospheric abundance of neodymium leading to a longstanding debate obtained from the study of Nd II lines due to a lack of atomic data such as gf-values. Suitable solar Nd II lines exist with accurate experimental gf-values is measured by Den Hartog et al. (2003), which permit us to determine the LTE solar photospheric abundance of neodymium.

The chemical composition of the solar photosphere has been studied by many investigators (Anders and Grevesse 1989; Grevesse and Sauval 1998; Cunha and Smith 1999; Asplund 2000; Lodders 2003; Asplund et al. 2005; Grevesse et al.2007; Shi et al. 2008; Lodderset al.2009; Asplund et al. 2009; Shchukina et al. 2012; Shaltout et al. 2013).Different results obtained from the solar photospheric abundance of Nd II lines are published in these papers by Ward et al. $1984\left(\log \varepsilon_{N d}=1.47 \pm 0.07\right)$, Ward et al. 1985 $\left(\log \varepsilon_{N d}=1.50 \pm 0.12\right)$ and Den Hartog et al. $2003\left(\log \varepsilon_{N d}=1.45 \pm 0.01\right)$.

2. The LTE theoretical Temperature model
The Solar model atmospheres are the main ingredient for providing the basic background of the spectral line formation. To obtain fundamental information about the sun's chemical composition from the solar spectrum, it is essential to have realistic models of the solar atmosphere. In the present work, the most widely semi-empirical solar model used for the abundance of $\mathrm{Nd}$ is the Holweger and Müller (1974, hereafter, HM) model, while our theoretical model is obtained under the assumption of LTE in radiative-convective equilibrium, where convection is treated in a mixing-length approach. The Atlas9 Fortran code written by kurucz (1993) is used to obtain the temperature model. For more details, our LTE theoretical temperature model is derived with the effective temperature Teff $=5777 \mathrm{~K}$, the surface gravity $(\mathrm{cm} / \mathrm{sec} 2) \log (\mathrm{g})=4.4377$ and the metallicity $\log [\mathrm{M} / \mathrm{H}]=0.0$. The solar photospheric abundances of all elements are adopted from Grevesse and Sauval (1998). The microturbulent velocity in the line opacity with $1.0 \mathrm{~km} \mathrm{~s}^{-1}$ is taken into account.Fig. (1) shows the comparison between our theoretical model (dotted line) with HM (solid line) solar model. This investigation assumes only the opacity source based on ODF, since it seems worthwhile extending our contribution to photospheric solar neodymium abundance between two atmospheric models.As evident from Fig. (1), we compare the two photospheric models in the range of optical depth between $\log \tau_{500}=1$ to -4.5 . The 


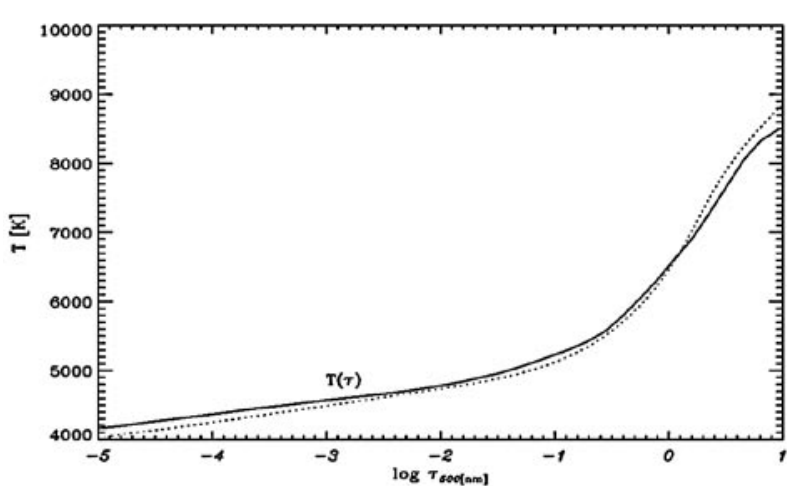

Fig. (1): Temperature stratifications of Opacity Distribution Function (ODF) (dotted line) and HM (solid line) solar models.

ODF (dotted line) solar model shows a small difference comparing with HM model between

$\log \tau_{500}=0$ to $\log \tau_{500}=1$. At some optical depth between $\log \tau_{500}=-1$ tolog $\tau_{500}=-2$, the ODF model is comparatively smaller in magnitude than the HM model with about a difference in temperature by $100 \mathrm{k}$. In the outer layers, the ODF model is slightly cooler than HM model.

\section{RESULTS AND DISCUSSION}

In the present work, we derive the solar photospheric abundance of Nd using two methods to confirm our results. The first approach assumes the LTE line formation with the spectrum synthesis code installed with FORTRAN language, which is called SYNTH program written by Kurucz (1993). The second approach is derived by fitting the measured equivalent width of single unblended lines to the computed equivalent width using WIDTH9 code, which is a FORTRAN program written by Kurucz (1993) for the determination of stellar abundances.

For the LTE line formation spectrum synthesis, only $23 \mathrm{Nd}$ II solar lines are used as well as the atomic data (excitation potentials, log gf values) is presented in Table (1). The first column gives the wavelength $(\mathrm{nm})$ of the individual lines, while the second column represents the

Table (1):A summary of line list selected for the solar Nd II lines used in the abundance determination. The 1D refers to one-dimensional temperature model:

\begin{tabular}{cccccccc}
\hline$\lambda[\mathrm{nm}]$ & $E_{1}[\mathrm{eV}]$ & $\log g f$ & $\gamma_{\mathrm{rad}}$ & $\gamma_{s}$ & $\gamma_{\mathrm{vd} w}$ & $\begin{array}{c}\log \epsilon_{\mathrm{Nd}}[1 \mathrm{D}] \\
{[\mathrm{HM} \text { model] }}\end{array}$ & $\begin{array}{c}\log \epsilon_{\mathrm{Nd}}[1 \mathrm{D}] \\
{[\text { Our model] }}\end{array}$ \\
\hline 361.581 & 0.2046 & -0.76 & 8.23 & -5.79 & -7.72 & 1.45 & 1.43 \\
378.424 & 0.3802 & 0.15 & 8.19 & -5.78 & -7.72 & 1.40 & 1.38 \\
383.898 & 0.0000 & -0.24 & 8.18 & -5.85 & -7.74 & 1.45 & 1.42 \\
390.022 & 0.4714 & 0.10 & 8.16 & -5.79 & -7.72 & 1.60 & 1.57 \\
399.010 & 0.4714 & 0.13 & 8.14 & -5.80 & -7.72 & 1.45 & 1.41 \\
402.133 & 0.3206 & -0.10 & 8.14 & -5.82 & -7.73 & 1.47 & 1.44 \\
406.108 & 0.2046 & 0.55 & 8.13 & -5.80 & -7.73 & 1.45 & 1.42 \\
440.082 & 0.0636 & -0.60 & 8.06 & -5.90 & -7.76 & 1.45 & 1.41 \\
444.638 & 0.3802 & -0.35 & 8.05 & -5.88 & -7.25 & 1.45 & 1.40 \\
454.260 & 0.7421 & -0.28 & 8.03 & -5.81 & -7.73 & 1.45 & 1.40 \\
456.322 & 0.1823 & -0.88 & 8.03 & -5.89 & -7.75 & 1.44 & 1.39 \\
470.654 & 0.0000 & -0.71 & 8.00 & -5.93 & -7.77 & 1.45 & 1.40 \\
470.972 & 0.1823 & -0.97 & 8.00 & -5.91 & -7.76 & 1.45 & 1.41 \\
471.559 & 0.2046 & -0.90 & 8.00 & -5.90 & -7.76 & 1.45 & 1.40 \\
477.772 & 0.3802 & -1.22 & 7.99 & -5.88 & -7.75 & 1.45 & 1.42 \\
478.611 & 0.1823 & -1.41 & 7.99 & -5.91 & -7.76 & 1.47 & 1.44 \\
482.034 & 0.2046 & -0.92 & 7.98 & -5.91 & -7.76 & 1.30 & 1.27 \\
509.279 & 0.3802 & -0.61 & 7.93 & -5.91 & -7.76 & 1.48 & 1.45 \\
513.059 & 1.3039 & 0.45 & 7.93 & -5.77 & -7.72 & 1.46 & 1.40 \\
525.551 & 0.2046 & -0.67 & 7.91 & -5.94 & -7.77 & 1.45 & 1.42 \\
527.343 & 0.6804 & -0.18 & 7.90 & -5.88 & -7.75 & 1.40 & 1.38 \\
529.316 & 0.8229 & 0.10 & 7.90 & -5.86 & -7.74 & 1.45 & 1.42 \\
531.981 & 0.5502 & -0.14 & 7.90 & -5.90 & -7.76 & 1.45 & 1.42 \\
& & & & & & 1.45 \\
\hline
\end{tabular}


atomic data of lower energy in the units of $(\mathrm{eV})$. The third column shows the multiplication of the statistical weight (g) and oscillator strength (f) in the logarithmic scale of gf-values which are taken from Den Hartog et al. (2003). The radiative, Stark and van der Waals damping constants are shown in the fourth $\left(\gamma_{r}\right)$, fifth $\left(\gamma_{s}\right)$, and sixth $\left(\gamma_{v w}\right)$ columns, respectively. While the last two columns exhibit the derived abundances from individual solar Nd II lines with HM and our theoretical models, respectively. For the equivalent width fitting method, we selected a good sample of 28 solar Nd II lines given in Table (2). As already discussed above, our explanations of the lines are the same as presented in Table (1), but in the fourth column the measured equivalent widths are shown. For more clarity, the damping parameters are not presented in Table (2). The atomic data for all of $27 \mathrm{Nd}$ II lines are taken from Den Hartog et al. (2003), but we select only one Nd II line with the wavelength of (584.239 $\mathrm{nm}$ ) from Kurucz database server.

From 23 solar Nd II lines, we derived Nd abundances from synthetic spectrum analysis. The selected lines are observed with FTS in the center of the solar disk. These lines are listed in the first column of Table (1); the individual abundance determined is shown in the last two columns. For the synthetic spectrum calculation, the present version of the LTE line analysis code SYNTH (Kurucz 1993) was employed. We adopted the solar model atmosphere of Holweger and Müller (1974), and a microturbulent velocity

Table (2): The LTE line list of singly ionized neodymium Nd II included in the abundance determination:

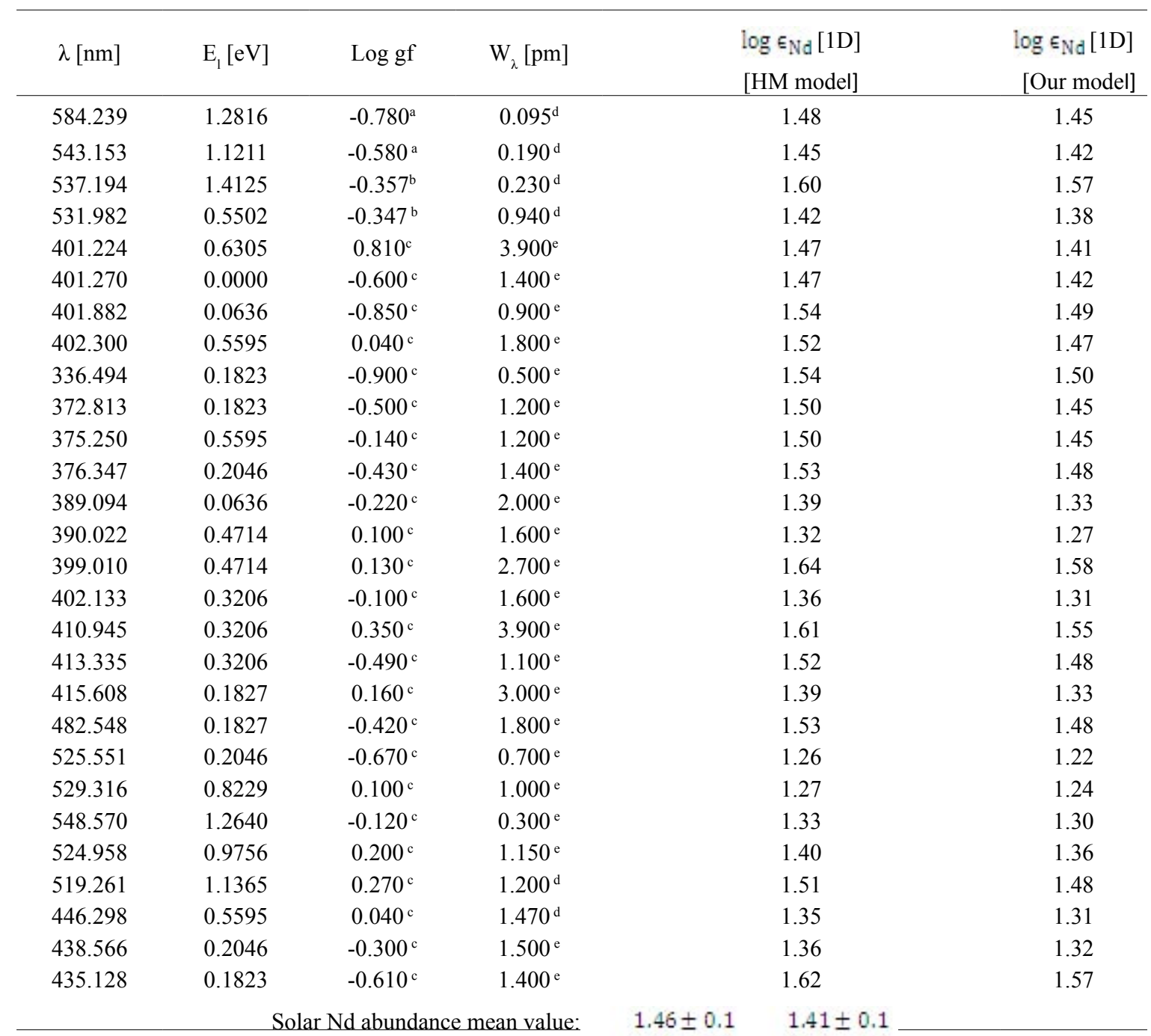

N.B: a: log (gf)-values measured by Ryder (2012). b: log (gf)-values measured by Ward et al.(1984). c: log (gf)-values measured by Den Hartog et al. (2003). d: Equivalent widthsare taken from Ward et al. (1984). e: Equivalent widths are taken from Moore et al. (1966). 
of $\xi=1.0 \mathrm{kms}^{-1}$ is used. Fig. (2) shows only two samples of synthetic spectrum matches to the $\mathrm{Nd}$ II lines and surrounding atomic and molecular features. When comparing the fitting line profiles obtained from the synthesis spectrum it was noticed that a large numbers of Nd II lines are less suitable for abundance determination. Also, the wings of $\mathrm{Nd}$ lines are not fitted well. It is essential to obtain quantum mechanical broadening treatment instead of empirical pressure broadening. Up to now, clearly the quantum broadening method is applied only for neutral lines of any chemical element, as already published in the series of papers such as the recipes of Anstee and O'Mara (1995), Barklem and O'Mara (1997) and Barklem et al. (1998). For more clarity, we summarized the reasons for the poor agreement in the line profile fitting. These reasons are: a) It is obvious that some of $\mathrm{Nd}$ II lines are suspected blends.
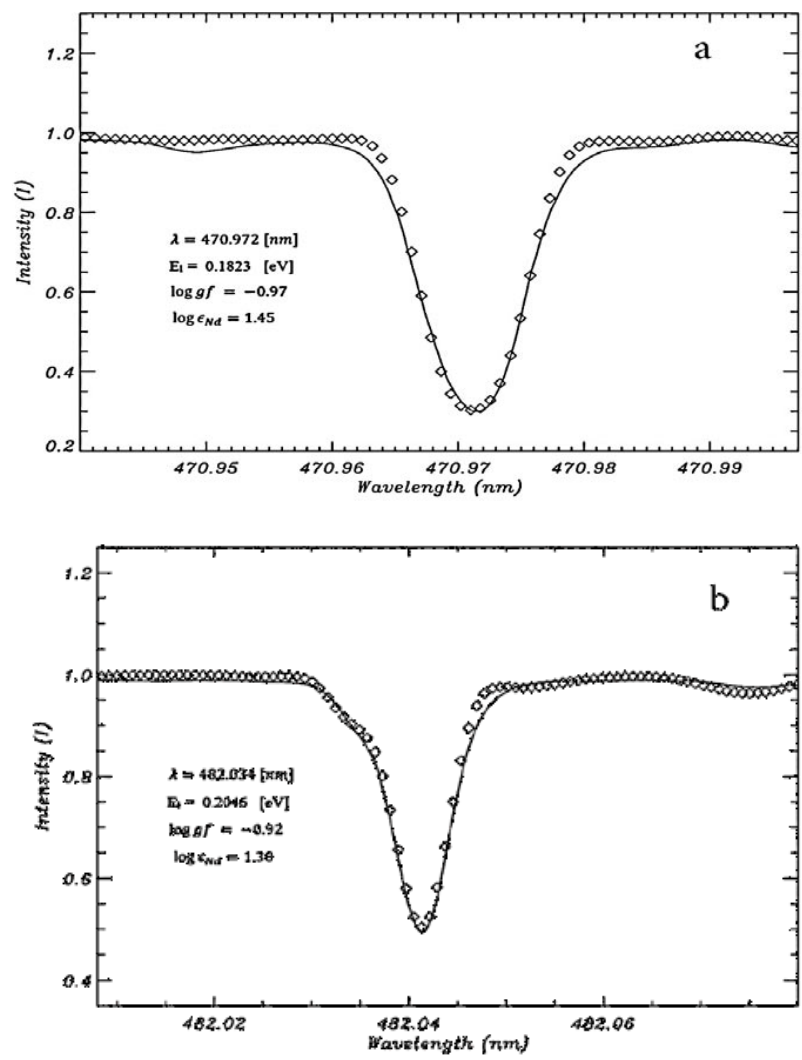

Fig. (2): The synthetic profiles of Nd II lines for $470.972 \mathrm{~nm}$ (a) and $482.034 \mathrm{~nm}$ (b)with (diamonds) compared with the observed Fourier Transform Spectrograph (FTS) profiles (solid lines). The synthetic profiles are obtained with HM solar model using the microturblent velocity of $\xi=1.0 \mathrm{~km} \mathrm{~s}^{-1}$ b) Due toa lack of atomic data of Nd II lines, it is expected that the damping parameters of Nd II lines affect the line profile fitting. The mean solar photospheric abundance with $\mathrm{HM}$ solar model from all $23 \mathrm{Nd}$ II solar lines is $\log \varepsilon_{N d}=1.44 \pm 0.04$. The derived $\mathrm{Nd}$ abundances with the wavelength of lines are shown in Fig. (3)(a) with a small change in the abundance values. The abundance obtained is not shown with the excitation potential because the total range is only $1.3 \mathrm{eV}$, so the range of excitation potential is rather small to show its variation with the derived abundance. Our result of $\mathrm{Nd}$ abundance obtained from our theoretical solar model is $\log \varepsilon_{N d}=1.41 \pm 0.04$ from all $23 \mathrm{Nd}$ II lines.
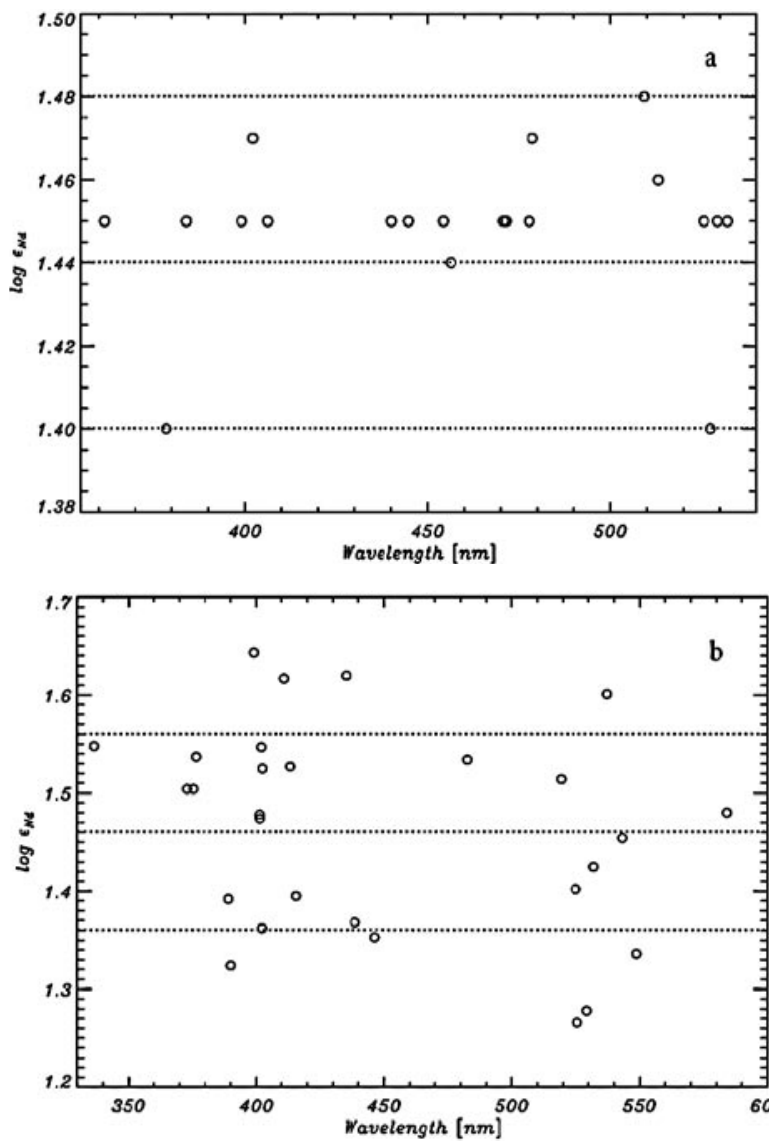

Fig. (3): The solar photospheric abundance of $\mathrm{Nd}$ II using two approaches.(a) The LTE singly ionized neodymium abundance values against 23 Nd II lines from synthetic spectrum analysisand (b) 28 Nd II lines using WIDTH9 code, wherethe derived abundance with the HM model using the microturblent velocityof . The horizontal lines illustrate the abundance mean and the standard deviation. 
The difference in the abundance of Nd between HM model and our model is $\Delta \xi=\xi(\mathrm{HM})-\xi$ (our model $)=0.03$ dex. It is attributed to the different sources of temperature models and neodymium lines selected.

For the second approach, we use the WIDTH9 Fortran program where the abundance of $\mathrm{Nd}$ II is derived by fitting the measured equivalent width of single unblended lines (of 28 lines) to the computed equivalent width. The measured equivalent widths of these lines are taken from Moore et al. (1966) and Ward et al. (1984). We derived the abundance using the $\mathrm{Nd}$ II lines as given in Table (2) with one dimensional solar Holweger and Müller (1974) and our theoretical models. Table (2) displays the final LTE abundance $(\varepsilon N d)$ obtained with a microturbulent velocity $\xi=1.0 \mathrm{~km} \mathrm{~s}^{-}-1$. The mean value for the solarphotosphericabundanceofneodymiumis $1.46 \pm 0.1$ with HM model from all $28 \mathrm{Nd}$ II lines, where
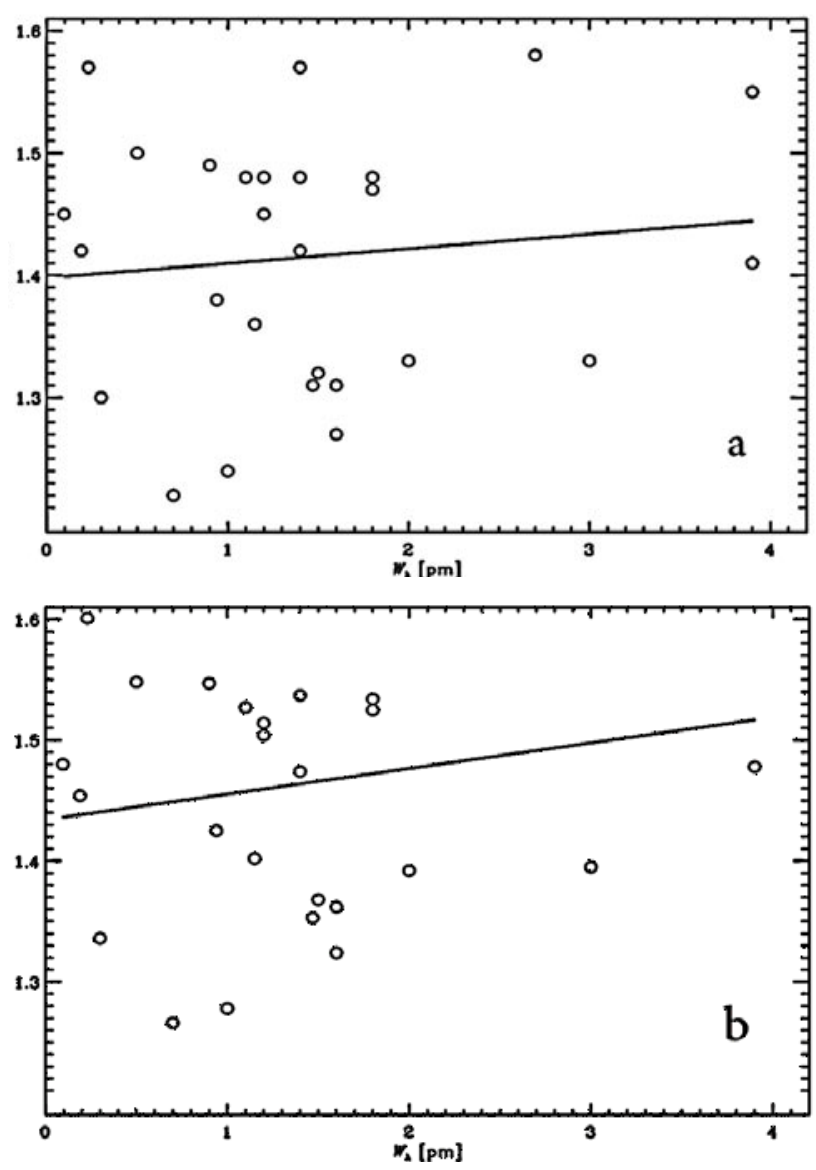

Fig. (4):The individual neodymium abundances derived from $28 \mathrm{Nd}$ II line profiles fitting of Nd II as a function of the equivalent width using HM model (a) and our theoretical model (b). the derived $\mathrm{Nd}$ abundances with the wavelength of lines are shown in Fig. (3) (b). Our result of Nd mean abundance obtained from our the oretical solar model is $\log \varepsilon_{N d}=1.41 \pm 0.04$ from all 28 Nd II lines. Fig. (4) shows the relation between the abundances derived from 28 solar lines as a function of equivalent width with HM model (a) and our model (b). It is clear that similar Nd abundance valuesare derived for the two solar models.

The mean solar photospheric neodymium abundance from all 51 solar lines is, where the derived $\mathrm{Nd}$ abundances with the wavelength of lines are shown in Fig. (5). This figure displays the values of $\mathrm{Nd}$ abundance (1.45), where the scatter is $( \pm 0.08)$ is attributed to many sources of gf-values. Asplund et al. (2009) determined the solarphotosphericabundance of Ndis $1.45 \pm 0.02$ using the 3D solar model. The meteoritic abundance value of $\mathrm{Nd}(1.45)$ is given more recently in the review of Asplund et al. (2009). The difference between our result $(1.45 \pm 0.08)$ and Den Hartog et al. (2003) $(1.45 \pm 0.01)$ is the microturbulent velocity adopted. Also, there is a difference in equivalent width between Den Hartog et al. (2003) and Moore et al. (1966).

\section{Conclusion}

The present paperpresentsanewdetermination of the solar Nd abundance by means of detailed LTE calculation using both

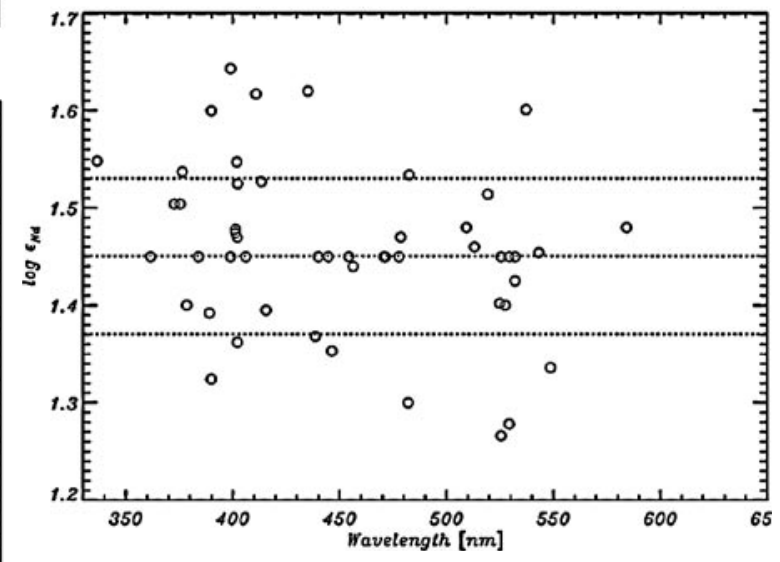

Fig. (5): The LTE singly ionized neodymium abundance values against 51 Nd II solar lines, where derived with the HM model usingthe microturblent velocity of $\xi=1.0 \mathrm{kms}^{-1}$. The horizontal lines illustrate the abundance mean and the standard deviation. 
a semi-empirical model (the venerable Holweger and Müller 1974 model) and our theoretical model of solar photosphere.

A new theoretical solar model of the photosphere is derived. Our theoretical model is slightly cooler than the HM model by $100 \mathrm{~K}$ in the outer layers.

In the present work, we have been used two methods for the abundance analysis. The first derived value of the abundance $(1.44 \pm 0.04)$ with HM model and our model $(1.41 \pm 0.04)$ using the synthetic spectral line profiles in comparing with the observed spectrum made by SYNTH FORTRAN (kurucz 1993). The second derived value of HM model $(1.46 \pm 0.1)$ and our model $(1.41 \pm 0.1)$ using the observed equivalent widths fitting in comparing with the calculated equivalent width with the WIDTH9 FORTRAN (kurucz 1993). The reasons for selecting two methods in the abundance determinations are, first, to feel confident about the result of solar neodymium abundance. Secondly, the synthetic spectrum fails to fit some solar Nd II lines owing to there exist the situation of blended lines with any specific line of another element in the solar spectrum region. Finally the mean solar photospheric abundance of neodymium is $(1.45 \pm 0.08)$ mostly the same abundance value of Den Hartog et al. (2003).

The solar photospheric abundance of neodymium using the experimental lifetime measurements are obtained from 51 solarNd II lines is $\left(\log \epsilon_{N d}=1.45 \pm 0.08\right)$, it agrees with the very accurate meteoritic data value. This confirms our new result of solar photospheric abundance of Nd. The resulting abundance is very similar to the value advocated by more recently of Asplund et al. (2009).

\section{REFERENCES}

Anders E.Grevesse N. 1989, Geochim. Cosmochim. Acta, 53,197-214 Asplund M. 2000, A\&A, 359, 755-58

Asplund M., Grevesse N., Sauval A. J. 2005, in Barnes T. G., III, Bash F. N., eds, ASP Conf. Ser. Vol. 336, Cosmic Abundance as Records of Stellar Evolution and Nucleosynthesis. Astron. Soc. Pac., San Francisco, p. 25-38

Asplund M., Grevesse N., Sauval A. J., Scott P. 2009 ARA\&A, 47,481

Anstee S. D., O’Mara B.J. 1995, MNRAS, 276, 859-66

Barklem P.S., O’Mara B.J. 1997, MNRAS, 290, 102

Barklem P.S., O’Mara B.J., Ross J.E. 1998, MNRAS 296, 1057

Cunha K. Smith V.V. 1999, ApJ, 512, 1006

Den Hartog E. A., Lawler J. E., Sneden C., Cowan J.J. 2003, the Astrophysical Journal, 148, 543-566

Grevesse N., Sauval A.J. 1998, Space Sci. Rev. 85, 161

Grevesse N., Asplund M., Sauval A.J. 2007, Space Sci. Rev., 130, 105

Holweger, H. and Muller, E. A. 1974, Solar Phys. 39, 19

Kurucz R. L. 1993, SAO KURUCZ CD-ROM, Nos. 13 and 18

Lodders K. 2003, ApJ, 591, 1220

Lodders K., Palme H., Gail H. P. 2009, Landolt-Bornstein, New Series in Astronomy and Astrophysics, in press. (arXiv: 0901.1149)

Moore, C. E., Minnaert, M. G. J., and Houtgast, J. 1966, the Solar Spectrum 2934 A to 8770 A (US NBS Monogr. 61; Washington: GPO)

Ryder, PhD physics thesis, Wayne State University, Michigan, 2012

Shchukina N. Sukhorukov A.,Trujillo Bueno J. 2012, ApJ, $755,176 \mathrm{~S}$

Shi J. R., Gehren T., Butler K., Mashonkina L. I. Zhao G., 2008, A\&A, 486, 303

Shaltout, A. M. K., Beheary M.M., Bakry A., Ichimoto K. 2013, MNARS, 430,2979S

Ward L., Vogel O., Arnesen A., Hallin R., McIntyre., Nordling C. 1984, 29, 551-555

Ward L., Vogel O., Arnesen A., Hallin R., Wännström A. 1985, PhysicaScripta., 31, 161-185 


\title{
ON USING ANFIS FOR RIVER NILE FLOW FORECASTING
}

\author{
Alaa M. A. Mahmoud* and S.A. Asklany** \\ Departments of Astronomy and Meteorology*, Al-Azhar University, Faculty of Science, Nasr City, Cairo 11884, \\ Egypt. Higher Technological Institute** (HTI), Next to Small Industries Complex, Industrial Area2,Tenth of \\ Ramadan City, AlSharqiya.
}

\begin{abstract}
An adaptive neuro-fuzzy inference system ANFIS is used in river Nile flow forecast by two different models; first model build on using precipitation only as input with the flow as output where in the second one we used precipitation and zonal wind over a specific area as inputs with the flow as output. Several years monthly mean precipitation data over catchments areas are used as input variable for the two models. In the models, the data sets were divided into the subsets training, checking, and testing data sets. In addition, we depend on ANFIS ability in chaotic time series prediction to predict the rivers flow for one month from the previous precipitation monthly mean data. The presented two models approved good performance with acceptable root mean square errors RMSE compared with classical statistical methods. All calculations were done under MATLAB environment.
\end{abstract}

Key words: Flood, forecast, Artificial Intelligence, Fuzzy, Neural networks, hydrology.

\section{INTRODUCTION}

Forecasting river flow is a key element in hydrology concerns e.g. managing water resources and flood control;precipitation with snow melting play champion ship role in supplying the rivers with fresh water. Where, evaporation, irrigation, water seeping toward underground water, and human activities are the sinks. Since that process built on many factors.

Varieties of traditional approaches were introduced to achieve water flow forecast such as linear regression and discriminate prediction; descriptions of those two techniques were introduced by $[15,1,9,16$, and 8$]$ used discriminate prediction approach for forecast. Parameterization approach recently introduced for river flow forecast; [19].

The artificial intelligent techniques are widely used in recent studies in the modeling and forecasting; [2] used ANFIS in seasonal prediction of ground water. In hydrological resources studies,[5] used adaptive neuro-fuzzy inference system for discharge modeling. [3]applied soft computing techniques in prediction of total bed material load in tropical rivers; [7]forecast the river flows using climatic forcing 2003.Also, [10] used neuro-fuzzy modeling and forecasting in water resources.

In this study, we investigate the capability of
ANFIS for modeling the river flow forecast.Two techniques were proposed, first we used precipitation data in ANFIS input layer to forecast monthly mean river flow for river Nile; adding the zonal wind area mean over a specific area in the ANFIS input layer the second model were built by using, two sets of data, training and checking. Many statistical parameters were used to assess the models.

\section{Data Description}

A variety of precipitation data sets were used in this study over three different areas: area one (A1) $00-5 \mathrm{~N}, 30-35 \mathrm{E}$, area2 (A2) $7.5-12.5 \mathrm{~N}$, 37.5-41.5E and area3(A3) $00-25 \mathrm{~N}, 25-40 \mathrm{E}$; the best correlation was found between the $\mathrm{A} 3$ precipitation data and the flow table 1 . The rainfall is over land the data source is IPCC (Intergovernmental Panel of Climate Change Data Distribution Center) CRU Ts3 data [14]. The data is distributed over land at a resolution 0.50 lat $\times$ $0.5 \mathrm{o}$ long for one hundred and two years data extended from January 1901 to December 2002 were used in this study.

The river flow data is the flow at Aswan, from August 1871 to July 2002 in billion cubic meters. These data are kindly given from the Ministry of Irrigation and Water Resources of the Arab Republic of Egypt (ARE).

The NCEP/NCAR Reanalysis project is us- 
ing a state-of-the-art analysis/forecast system to perform data assimilation using past data from 1948 to 2002. A subset of this data has been processed to create monthly means of a subset of the original data. There are also files containing data from variables derived from the reanalysis and some other statistics.

These are calculated by averaging the 4 times daily data into monthly means. In some cases, the units were changed from the original NCEP data. Means are generally from 1948-2002 though some variables are available for a subset of that period.The variable used is the zonal wind $\mathrm{m} / \mathrm{s} . \mathrm{NCEP} / \mathrm{NCAR}$ Reanalysis Monthly Means for the zonal wind over the area $00^{\circ}-30^{\circ}$ $\mathrm{N},-60^{\circ}-70^{\circ} \mathrm{E}$ fig. 1 .

\section{METHOD}

As system complexity increases, reliable fuzzy rules and membership functions used to describe the system behavior are difficult to determine. Use of ANFIS has been published in several journals for various forecasting applications to overcome these difficulties. Neuroadaptive learning techniques provide a method to integrate information from a data set (learning process), in order to compute the membership function parameters that best allow the associated fuzzy inference system to track the given input/ output data [13]. Back propagation is used to modify the initially chosen membership functions and the least mean square algorithm determines the coefficients of the linear output functions [20]. A neural network which can perform pattern matching task has a large number of highly interconnected processing elements (Nodes).

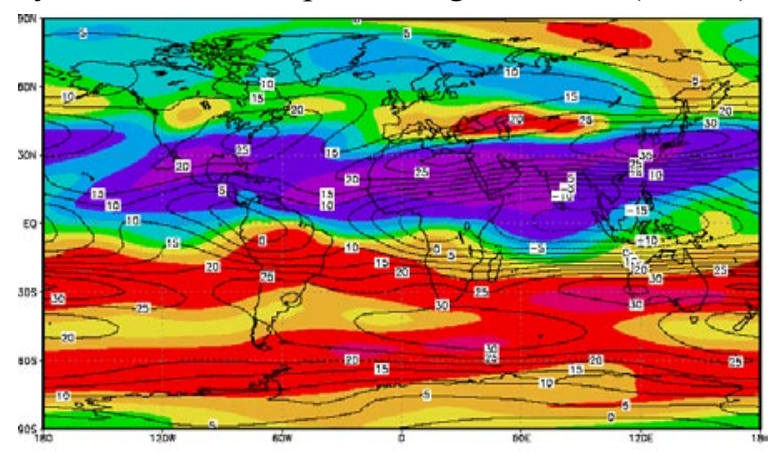

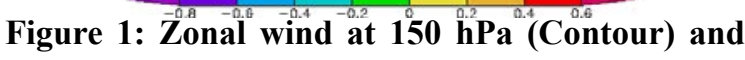
its correlation coefficient (Shaded) with the flood at Aswan.
These elements demonstrate the ability to learn and generalize from training patterns. Distributed representation and strong learning capabilities are the major features of neural network. On the other hand, decisions using fuzzy logic systems are based on inputs in the form of linguistic variables. These linguistic variables are derived from membership functions which are formulas used to determine the fuzzy set to which a value belongs and the degree of membership in that set. The variables are then matched with the specific linguistic IF-THEN rules and the response of each rule is obtained through fuzzy implication. To perform compositional rule of inference, the response of each rule is weighted according to the values or degree of membership of its inputs and the centroid of response is calculated to generate the appropriate output. Neural network has the shortcoming of implicit knowledge representation. However, fuzzy logic systems are subjective and heuristic. These drawbacks of neural network and fuzzy logic systems are overcome by the integration between the neural network technology and the fuzzy logic systems; so the ANFIS could be viewed as a fuzzy system, a neural network or fuzzy neural network. The specific advantages of ANFIS over the two parts of this hybrid system are:

i. ANFIS has the ability to classify data and find patterns.

ii. ANFIS develops a fuzzy expert system that is more transparent to the user and also less likely to produce memorization errors than a neural network.

Furthermore, ANFIS keeps the advantages

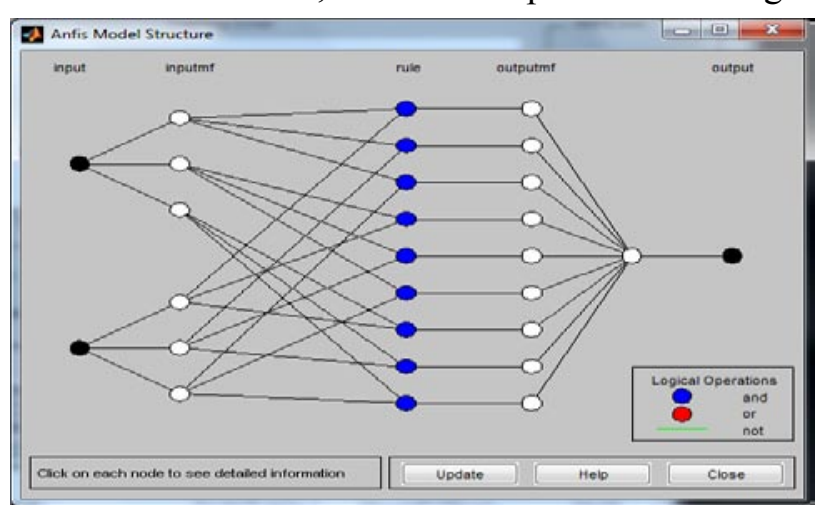

Figure 2: ANFIS structure. 
of a fuzzy expert system, while removing (or at least reducing) the need for an expert.

ANFIS [20]implements Takagi-Sugeno fuzzy rules in five layers. For simplicity, it is assumed that the fuzzy inference system under consideration has two inputs $\mathrm{x}$ and $\mathrm{y}$ and one output $\mathrm{z}$ as shown in Figure 2.

For a zero-order Sugeno fuzzy model, a common rule set with two fuzzy if-then rules is the following:

Rule 1: If $\mathrm{x}$ is $\mathrm{A} 1$ and $\mathrm{y}$ is $\mathrm{B} 1$, Then $\mathrm{f} 1=\mathrm{r} 1$

Rule 2: If $\mathrm{x}$ is $\mathrm{A} 2$ and $\mathrm{y}$ is $\mathrm{B} 2$, Then $\mathrm{f} 2=\mathrm{r} 2$

Here the output of the ith node in layer $\mathrm{n}$ is denoted as $\mathrm{O}_{\mathrm{n}, \mathrm{i}}$ :

i. The first layer represents fuzzy membership functions.

Every node $\mathrm{i}$ in this layer is an adaptive node with a node function:

$$
\begin{array}{ll}
\mathrm{O}_{1, \mathrm{i}}=\mu \mathrm{A}_{\mathrm{i}}(\mathrm{x}) & \text { for } \mathrm{i}=1,2,3 \text { or } \\
\mathrm{O}_{1, \mathrm{i}}=\mu \mathrm{B}_{\mathrm{i}-3}(\mathrm{y}) & \text { for } \mathrm{i}=4,5,6(4)
\end{array}
$$

Where $\mathrm{x}$ (or $\mathrm{y}$ ) is the input to node $\mathrm{i}$ and $\mathrm{A}_{\mathrm{i}}$ $\left(\operatorname{orB}_{\mathrm{i}}\right)$ is a linguistic label associated with this node. In other words, O1,Iis the membership grade of a fuzzy set $\mathrm{A}_{1}, \mathrm{~A}_{2}$ and $\mathrm{A}_{3}$ (or $\mathrm{B}_{1}, \mathrm{~B}_{2}$ and $\mathrm{B}_{3}$ ) and it specifies the degree to which the given input $\mathrm{x}$ (or $\mathrm{y}$ ) satisfies the quantifier $\mathrm{A}$ (or B). Here the membership function for A (or B) is triangular membership.

ii. The second and the third layers contain nodes that form the antecedent parts in each rule. Every node in the layer two is a fixed node whose output is the product of all the incoming signals:

$$
\mathrm{O} 2,_{\mathrm{i}}=\mathrm{w}_{\mathrm{i}}=\mu \mathrm{A}_{\mathrm{i}}(\mathrm{x}) \mu \mathrm{B}_{\mathrm{i}}(\mathrm{y}) \quad \mathrm{i}=1,2,3
$$

Each node output represents the firing strength of a rule. In layer three every node $i$ in this layer is an adaptive node with a node function:

$$
\underset{\mathrm{i}=1,2,3}{\mathrm{O}_{3, \mathrm{i}}}=\mathrm{w}_{\mathrm{i}} \quad \mathrm{f}_{\mathrm{i}}=\text { wiri }
$$

Where $\mathrm{i}$ is the parameter set of this node. Parameters in this layer are referred to as consequent parameters and $\mathrm{w}$ is the base-width. iii. The fourth layer calculates the first-order Takagi- Sugeno rules for each fuzzy rule.

iv. The fifth layer- the output layer, calculates the weighted global output of the system.

$$
O_{5 i}=\frac{\sum_{i} w_{i} f_{i}}{\sum_{i} w_{i}}
$$

Where $O_{s i}$ the global output and $\mathrm{W}$ is the base-width [17].

\section{RESULTS AND DISCUSSION}

Two models were built depend on our data set; first we divided the data into three sets one for training and one for testing and the last one for checking the models. Normalization to the data vector to be between 0 and 1 because of the parameters used have different scales and units, by using the following formula:

$$
X_{\text {norm }}=\frac{x-x_{\min }}{x_{\max }-x_{\min }}
$$

In the first model only one input parameter were used which is the precipitation and one output to the model which is flood. The process of edit the ANFIS by aid of MATLAB fuzzy toolbox by loading training and checking data sets then generate FIS assign three triangular membership functions to the input parameter fig. 3 .

The triangular MFs:

$$
f(x, a, b, c) \max \left(\min \left(\frac{x-a}{b-a}, \frac{c-x}{c-b}\right), 0\right)
$$

Where a and bare the premise parameters that characterize the shapes of the input MFs.

After built the model and get the flood calcu-

Table 1: The correlation coefficient between precipitation and Nile river flow at Aswan for three different areas, area $1(00-5 \mathrm{~N}, 30-35 \mathrm{E})$, area $2(7.5-12.5 \mathrm{~N}, 37.5-41.5)$, area $3(00-25 \mathrm{~N}, 25-$ 40E).

\begin{tabular}{cccc}
\hline $\begin{array}{c}\text { Time } \\
\text { delayed }\end{array}$ & Area 1 & Area 2 & Area 3 \\
\hline One month & 0.41 & 0.83 & 0.8 \\
Two month & 0.39 & 0.71 & 0.74 \\
$\begin{array}{c}\text { Three } \\
\text { month } \\
\text { Four } \\
\text { month }\end{array}$ & 0.43 & 0.38 & 0.53 \\
\hline
\end{tabular}


Alaa M. A. Mahmoud and S.A. Asklany

lation we tested the model by calculating the root mean square Fig. 4 error, bias, correlation coefficient and absolute error table 2. Another model we built using two inputs: precipitation and wind also examine the model by the same manner we found that when wind is added as input the error generally reduced Fig.5. The models were used to forecast the river flow then the results were tested against the actual data Fig.6 and Fig.7.

Table 2: Statistical parameters for model 1 and model2

\begin{tabular}{ccc}
\hline & $\begin{array}{c}\text { Without } \\
\text { Wind } \\
\text { (model1) }\end{array}$ & $\begin{array}{c}\text { With Wind } \\
\text { (model2) }\end{array}$ \\
\hline $\begin{array}{c}\text { Correlation } \\
\text { bias } \\
\text { Mean Absolute } \\
\text { Error } \\
\text { Root Mean Square } \\
\text { Error }\end{array}$ & 0.88 & 0.95 \\
\hline
\end{tabular}

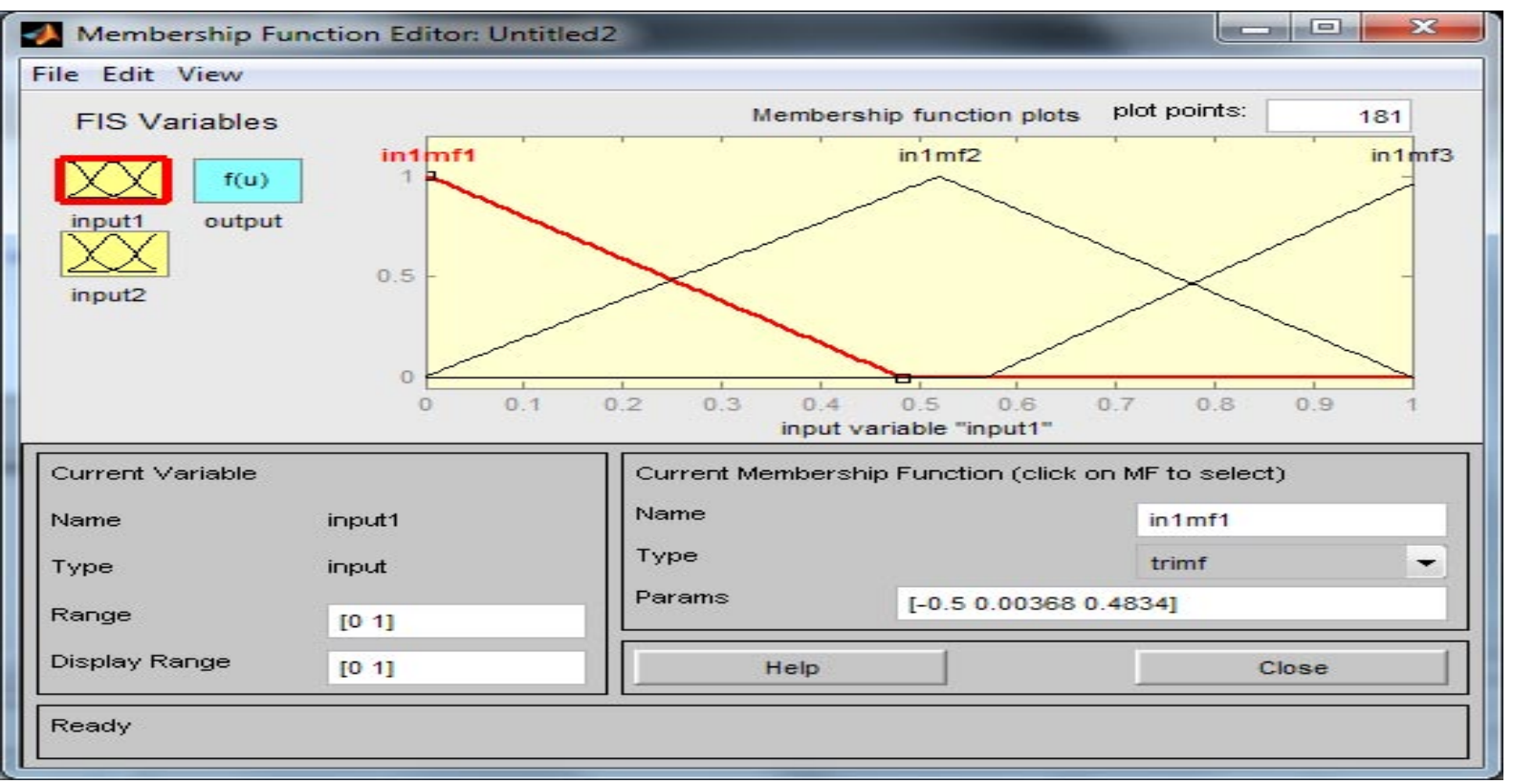

Figure 3: Triangular membership function.

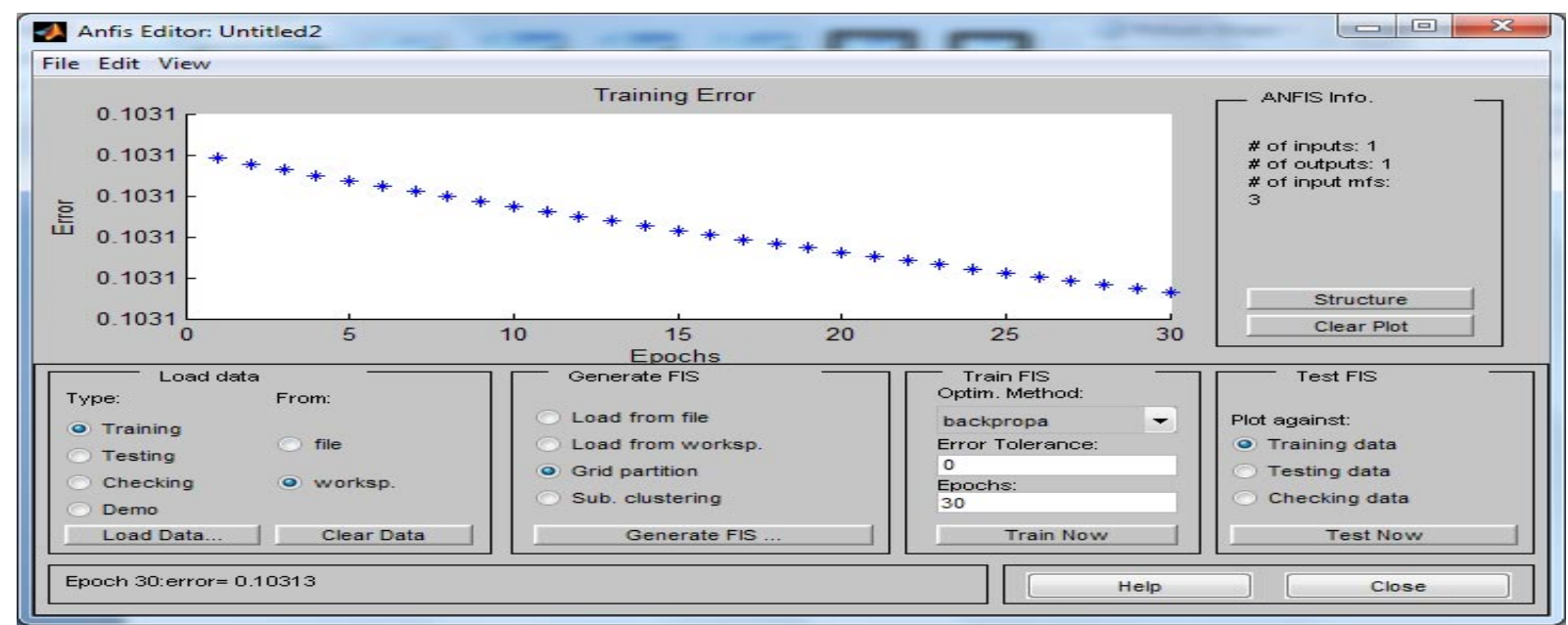

Figure 4: Error when precipitation only used as input. 


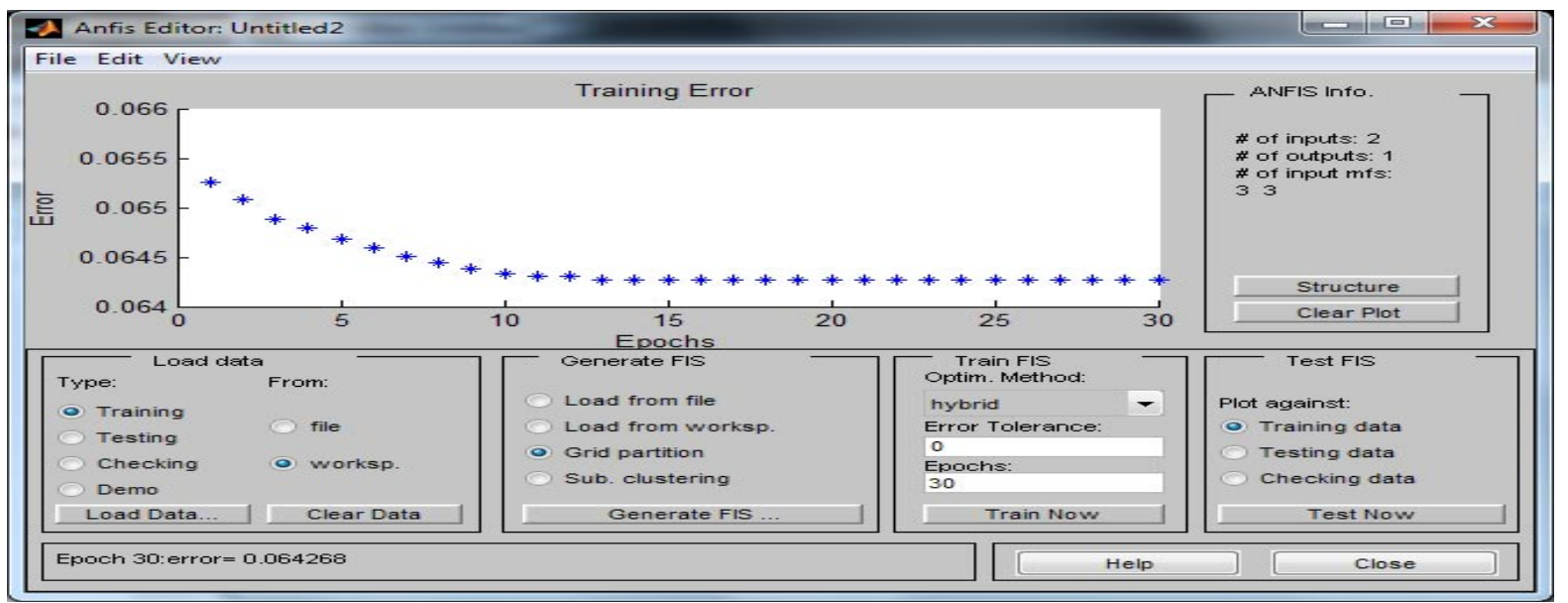

Figure 5: Error when precipitation and zonal wind used as inputs.

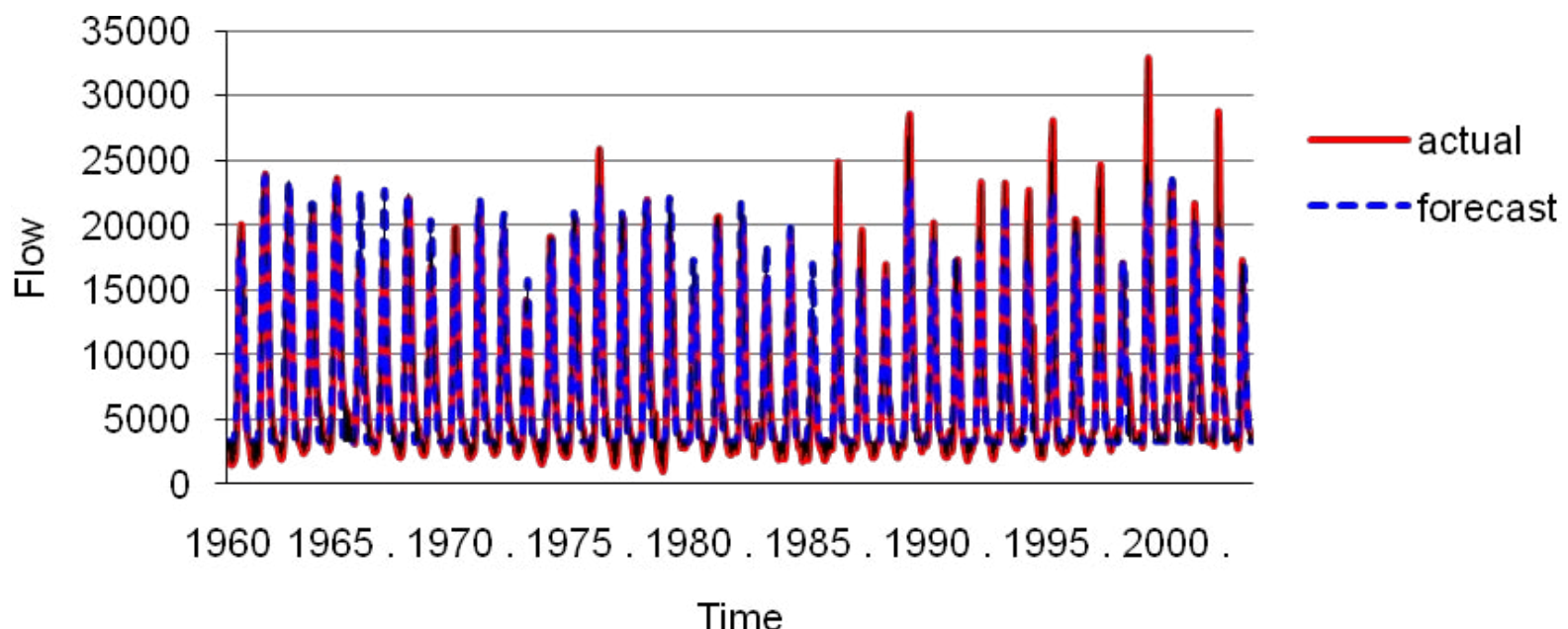

Figure 6: Actual and forecast data of model 1.

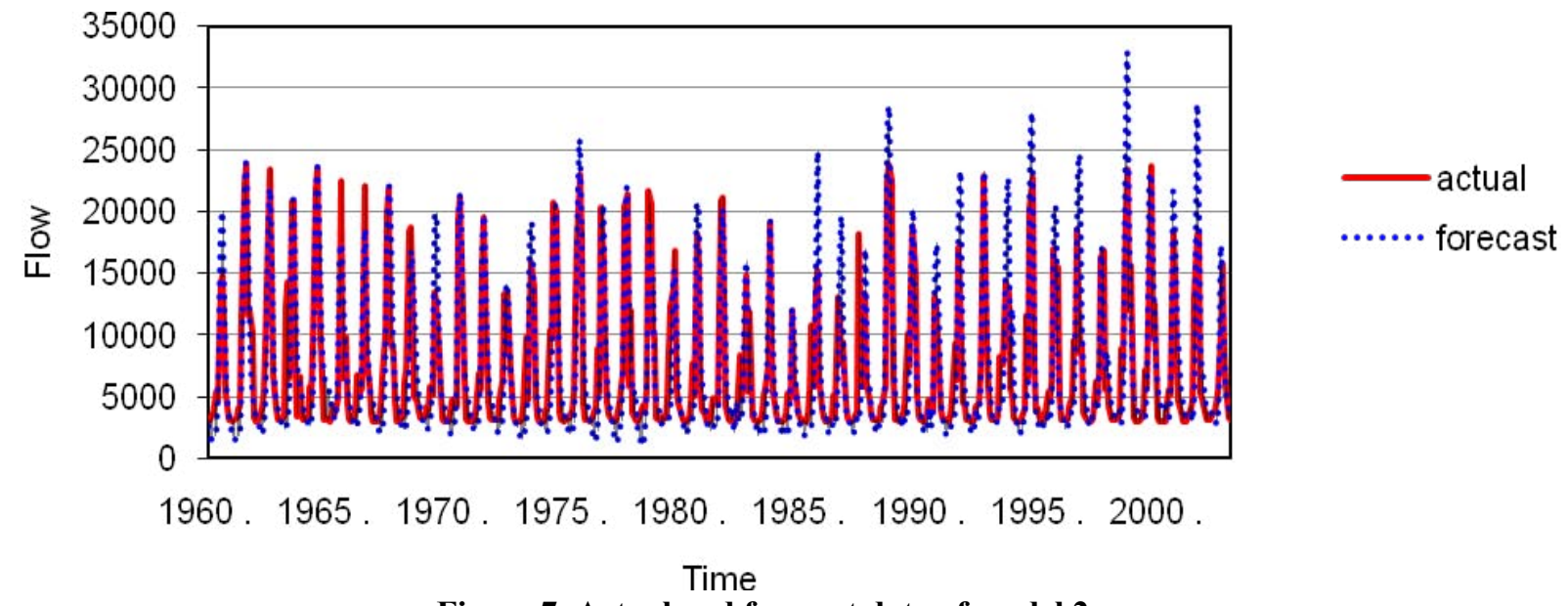

Figure 7: Actual and forecast data of model 2. 


\section{Conclusion}

Results agree with observations very well. Not only precipitation but also many meteorological parameters may used to improve the flood prediction like zonal wind. In this study, an adaptive neuro-fuzzy inference system is used to build two models to forecast flood flow. Even though FIS models trained usually have very good forecasting ability, their performance is not ideal when applying to predict variable like flood, which has chaotic behavior. This raises the possibility that fuzzy logic models could be further improved so they should not only be able to represent frequently occurring relationship but also be able to update itself like neural networks in learning possibility; so using ANFIS is very ideal in our case. The needs to background knowledge that will allow model to reinterpret and/or combine concepts in the data into new concepts that can lead to more accurate and/or simpler patterns. That is the direction for the soft-computing study which applied in our models and approved very good performance.

\section{ACKNOWLEDGMENTS}

NCEP Reanalysis Derived data are provided by the NOAA-ESRL Physical Sciences Division, Boulder Colorado from their Web site at http://www.esrl.noaa.gov/psd/.

\section{REFERENCES}

[1]Afifi, A. A., S.P.Azen 1979: Statistical Analysis- A Computer Oriented Approach. Academic Press, pp 442

[2] Amutha R., Porchelvan P.2011: Seasonal prediction of groundwater levels using ANFISand radial basis neural network, International Journal of Geology, Earth and Environmental Sciences ISSN: 2277-2081, 1,98-108

[3] ChangC K, H MdAzamathulla, N AZakaria and A AbGhani, 2012: Appraisal of soft computing techniques in predictionof total bed material load in tropical riversJ. Earth Syst. Sci.121,125-135

[4] Dai, A., T. Qian, K. E. Trenberth, and J.D. Milliman 2009: Changes in continental freshwater discharge from 1948-2004, J. Climate, 22, 2773-2792.

[5] Dinesh C. S. Bisht and Ashok Jangid ,2011: Discharge Modeling using Adaptive Neuro - Fuzzy Inference System, International Journal of Advanced Science and Technology, 31,99-114

[6] El-Shafie, A., and A. Noureldin 2011: Generalized versus non-generalized neural network model for multilead inflow forecasting at Aswan High Dam. Hydrol.
Earth Syst. Sci.,15, 841-858, doi:10.5194/hess-15-8412011.

[7] Eldaw, K., 2003: Long-Range Forecasting of the NileRiver Flows Using Climatic Forcing. J. of Appl. Meteor., 42, 890-904.

[8] Eltahir, E. A., 1996: El Niño and the natural variability in the flow of the NileRiver. WaterResource. Res., 32, 131-137.

[9] Folland CK, J. Owen, N. Ward, A., Colman, 1991: Prediction of seasonal rainfall in the Sahel region using empirical and dynamical methods. Journal of forecasting, 10, 21-56.

[10] HadiGalavi and Lee TeangShui 2012: Neuro- fuzzy modeling and forecasting in water resourcesSci. Res. Essays, 7(24), 2112-2121.

[11] Harris, I., Jones, P.D., Osborn, T.J. and Lister, D.H. (2014), Updated high-resolution grids of monthly climatic observations - the CRU TS3.10 Dataset. International Journal of Climatology 34, 623-642 doi:10.1002 joc.3711

[12] Jang, J.S.R.,1993. ANFIS: Adaptive-Network-Based Fuzzy Inference Systems. IEEE Transactions on Systems, 23, No. (3), 665-685.

[13] MasoudHessami, François Anctil and Alain A. Viau,2003: An adaptive neuro-fuzzy inference system for the post-calibration of weather radar rainfall estimation, Journal of Hydroinformatics, 5, 63-70

[14] Mitchell TD, Jones PD. 2005. An improved method of constructing a database of monthly climate observations and associated highresolution grids.Int. J. of Clim.25: 693-712.

[15] Parsons, B., McKenzie, D., 1978. Mantle convection and the thermal structureof the plates.J.Geophys. Res.,83, 4485-4496.

[16] Simpson, H. J., M. A. Cane, S. K. Lin, and S. E. Zebiak, 1993:. Forecasting annual discharge of River Murray, Australia, from a geophysical model of ENSO. J. Climate6:386-387.

[17] Tamer S. Kamel M. A. Moustafa Hassan, 2009: OnLine Trained Adaptive Neuro-Fuzzy Inference System for Distance Relay of Transmission line Protection, The Online Journal on Electronics and Electrical Engineering (OJEEE), 1, No. (2), 73-77.

[18] Wang, G., and E. A. B. Eltahir, 1999: Use of ENSO information in medium- and long-range forecasting of the Nile floods. J. Climate, 12, 1726-1737

[19] XubinZeng, Kira L. Kiviat, KoichiSakaguchi, Alaa M.A. Mahmoud 2012: A toy model for monthly river flow forecasting, J. of Hydrology452-453.226-231.

[20] Yanbo Huang 2009: Advances in Artificial Neural Networks - Methodological Development and Application Algorithms, 2, pp. 973-1007. 